\title{
Fabrication and characterization of a novel photoactive- based (0-3) piezocomposite material with potential as a functional material for additive manufacturing of piezoelectric sensors
}

\author{
O. A. Omoniyi ${ }^{1, \star}$, R. Mansour ${ }^{1}$ (D, M. J. Cardona ${ }^{1}$, M. L. Briuglia ${ }^{1}$, R. L. O'Leary ${ }^{1}$, and \\ J. F. C. Windmill ${ }^{1}$ \\ ${ }^{1}$ Centre for Ultrasonic Engineering, Electronic \& Electrical Engineering Department, Bioacoustics Group, University of Strathclyde, \\ Glasgow, Scotland, UK
}

Received: 29 July 2020

Accepted: 22 March 2021

Published online:

20 April 2021

(C) The Author(s) 2021

\begin{abstract}
The development of 3D-printed sensors and actuators from piezocomposite materials has increased in recent years due to the ease of production, low-cost and improved functionality additive manufacturing provides. The piezocomposite material developed in this work has the potential to be used as a functional material in stereolithographic additive manufacturing by combining the optical, viscoelastic properties of NOA 65 and the piezoelectric properties of Barium Titanate. The new (0-3) piezocomposite material consists of Norland Optical Adhesive 65 (NOA 65) as the polymer matrix and Barium Titanate $\left(\mathrm{BaTiO}_{3}\right)$ with particles sizes $(100 \mathrm{~nm}, 200 \mathrm{~nm}$ and $500 \mathrm{~nm})$ as the dielectric filler. We synthesized thin film samples of the (0-3) piezocomposite with $60 \%$ $\mathrm{w} / \mathrm{w} \mathrm{BaTiO}_{3}$ using solution mixing and spin coating method to produce samples with layer thickness of $100 \mu \mathrm{m}$. Fourier-transform infrared spectroscopy (FTIR) and Scanning electron microscopy (SEM) techniques were used to analyze the microstructure of the piezocomposite to determine the effect of different particles sizes of $\mathrm{BaTiO}_{3}$ on the structural and mechanical properties of the composite. The longitudinal piezoelectric coefficient $d_{33}$ was also measured using the laser vibrometer technique. Both single point scans and full surface scans were carried out to obtain the average piezoelectric coefficient $d_{33}$ of the composite material. The results of the SEM confirmed the (0-3) structure of the piezocomposite material with isolated $\mathrm{BaTiO}_{3}$ nanoparticles. It further showed the uniform distribution of the $\mathrm{BaTiO}_{3}$ nanoparticles across each of the samples. FTIR analysis showed that the filler nanoparticles had no effect on the native structure of the polymer matrix. The longitudinal piezoelectric coefficient $d_{33}$ of the piezocomposite material was observed to increase with increasing $\mathrm{BaTiO}_{3}$
\end{abstract}

Address correspondence to E-mail: Oluwaseun.omoniyi@strath.ac.uk 
particle sizes, while the indentation modulus of the composite investigated using the method of Oliver and Pharr was observed to decrease with an increase in particle size. Results from the single point scans showed the composite with $\mathrm{BaTiO}_{3}$ particle size $100 \mathrm{~nm}, 200 \mathrm{~nm}$ and $500 \mathrm{~nm}$ having an average $d_{33}$ of $2.1 \mathrm{pm} / \mathrm{V}, 3.0 \mathrm{pm} / \mathrm{V}$ and $3.9 \mathrm{pm} / \mathrm{V}$ while the average $d_{33}$ obtained from the full surface scan of $1430 \mathrm{scan}$ points showed $1.4 \mathrm{pm} / \mathrm{V}, 6.1 \mathrm{pm} / \mathrm{V}, 7.2 \mathrm{pm} / \mathrm{V}$.

\section{Introduction}

Piezocomposite materials with excellent mechanical and piezoelectric properties are increasingly being developed to be used in additive manufacturing of new sensors and actuators which are highly efficient, lightweight, and cheap. Additive manufacturing technology offers a wider degree of geometric freedom, the possibility of combining different materials for complex functions to easily meet fabrication needs [1]. Developing sensing and actuating applications from piezocomposite materials via additive manufacturing relies on the combination of materials of different phases such as polymers and ceramic nanoparticles. This process involves dispersing ceramic nanoparticles within a photolabile polymer resin to form a piezocomposite mixture with the $(0-3)$ connectivity pattern. This method is therefore used to create functional applications derived from the combination of the most useful properties of each constituent material within the bulk such as the piezoelectric sensitivity of the ceramic and the flexibility of the polymer.

Previously, piezocomposite materials have been widely used in the manufacture of electromechanical transducers such as pressure sensors, hydrophones, vibration sensors and many more [2]. These have mainly comprised of lead zirconate titanate (PZT) or barium titanate (BT) as the dielectric fillers due to their excellent piezoelectric properties and thermoplastic elastomers, and epoxy as the polymer matrix. Piezocomposite materials with different connectivity patterns have been described in [3] and are classified according to the way both phases are connected dimensionally e.g., (1-, 2-, or 3-) into 10 structures; $0-0,0-1,0-2,0-3,1-1,1-2,1-3,2-2,2-3$, and 3-3 [3]. One such pattern which offers much promise in the fabrication of highly functional transducers is the piezocomposite with (0-3) connectivity pattern.
The (0-3) composite was first discovered by Kitayama Pauer and Sugawara [4,5], using PZT as the filler material and polyurethane as the polymer matrix, $\mathrm{PBTiO}_{3}$ ceramic and eccogel polymers, $\mathrm{PBTiO}_{3}$ powder and chloroprene rubber. In a composite with (0-3) connectivity, ceramic nanoparticles are dispersed randomly within polymer phase. In this pattern, the ceramic particles are not in contact with each other. These composites can be used to easily fabricate large flexible thin sheets, produce applications in large scale and shaped to conform to variety of shapes [6]. Transducers for sonar applications have been fabricated using the $(0-3)$ piezoelectric composite due to their ease of manufacture at large scale and inherent flexibility [7]. Further application of this composite is its use as piezoelectric paint, and for damping mechanical vibrations [7]. And more recently, additive manufacturing of a fully 3D-printed piezoelectric microphone [8].

Recently, piezocomposite materials that have been used in 3D-printing of functional applications, includes materials consisting of BT, and Poly ethylene glycol diacrylate (PEGDA) [8], BT and bisphenol-A ethoxylate dimethacrylate (BEMA) [9] with [10] making use of modified BT was the most commonly used for additive manufacturing due to the high natural piezoelectric response of BT and photoactive property of PEGDA. However, such (0-3) piezocomposite material exhibits low piezoelectric response, with $d_{33}$ coefficients two orders of magnitude lesser than that of piezoelectric ceramic and single crystals [11]. Obtaining higher $d_{33}$ coefficients by increasing the concentration of the ceramic nanoparticles trades off the ease of processability of the piezocomposite during printing. A high particle concentration often results in a composite with high viscosity and also significantly increases attenuation of light through the material due to scattering from the particles. This often inhibits the ability to form intricate geometries and considerably reducing functionality of the printed structure [12, 13]. 
Barium titanate has been widely studied over the years. It is a typical perovskite-type ferroelectric material that is widely used in contemporary devices due to its well-balanced ferroelectric and high natural piezoelectric responses and favorable dielectric constant [14]. Although it has been shown that under specific arrangements, using BT nanotubes or rods greatly affects it ferroelectric properties with BT nanoparticles giving higher $d_{33}$ values [14].

In this work, we focus on the implementation of a (0-3) piezoelectric ceramic-polymer composite comprising BT and an UV curable adhesive, Norland Optical Adhesive 65 (NOA 65). NOA 65 is a clear, colorless, liquid photopolymer that will polymerize when exposed to ultraviolet light with a maximum absorption within the range of $350-380 \mathrm{~nm}$-the cured adhesive is very flexible with a modulus of elasticity of $10 \mathrm{MPa}$ and can be easily molded to different shapes [15]. Furthermore, the processing methods employed are described in detail along with the structural, mechanical and electrical properties of the resultant composites. Spin coating technique has been shown to be a versatile, inexpensive and highly effective technique for depositing uniform thin films [16] and extensively used to fabricate solution-processed organic/inorganic electronic devices [17-19]. This technique was also employed in this work to obtain uniform thin film samples.

\section{Materials and methods}

The materials used to fabricate the (0-3) piezocomposite include commercially available Norland Optical Adhesive 65 Polymer (Norland Products INC, NJ USA), and BT $100 \mathrm{~nm}, 200 \mathrm{~nm}$ and $500 \mathrm{~nm}$ nanoparticles (US research nanomaterials INC, TX USA). The NOA 65 material was used as supplied. The BT material underwent ball milling using $20 \mathrm{~mm}$ diameter alumina balls for a period of $24 \mathrm{~h}$, to deagglomerate the BT nanoparticles prior to preparing the piezoelectric composite mixtures. The process of developing the material is shown in Fig. 1

\subsection{Material formulation and spin coating}

A composite consisting of a ceramic filler with different nanoparticle sizes and a UV curable polymer material was used to make the samples reported in this work. The polymer matrix used had a viscosity of

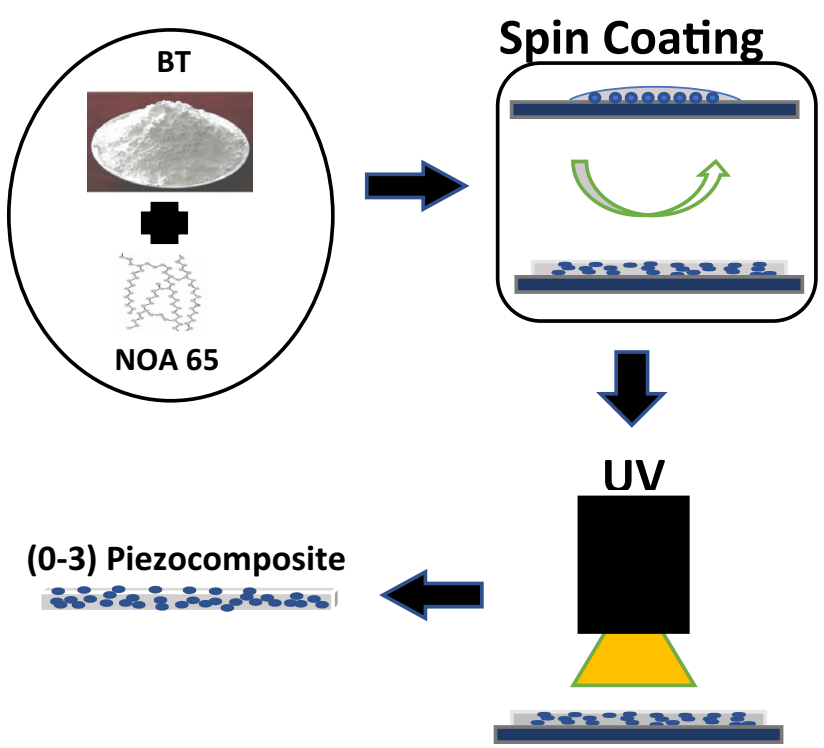

Fig. 1 Shows the process flow used in making the samples. It includes the solution mixing, spin coating and curing process

$1200 \mathrm{cps}$ at $25{ }^{\circ} \mathrm{C}$ making it possible to obtain higher filler loading up to $60 \% \mathrm{w} / \mathrm{w}$.

The piezoelectric composites were prepared by mixing the ball milled $\mathrm{BT}$ at $60 \% \mathrm{w} / \mathrm{w}$ with the NOA65 at $40 \% \mathrm{w} / \mathrm{w}$ using a THINKY AER-250 planetary mixer (Intertronics, Oxfordshire, England). This ratio was chosen because a higher loading of BT above $60 \% \mathrm{w} / \mathrm{w}$ resulted in a highly viscous mixture. This is due to the high $\mathrm{w} / \mathrm{w} \%$ fraction of the BT nanoparticle not embedding in the NOA 65 chain. The resulting mix was difficult to spin coat and cure under UV light even after leaving the samples over $24 \mathrm{~h}$. Furthermore, a lower solid loading less than $50 \% \mathrm{w} / \mathrm{w}$ BT loading resulted in very low piezoelectric properties since the nanoparticle sizes influences the mechanical and electrical properties of the composite material.

In order to prepare the thin film samples, an Ossila Spin coater (Ossila Ltd, Sheffield, UK) was used with the following process flow. A part of the BT composite mixture was spin coated on a glass slides at $2000 \mathrm{rpm}$ for $10 \mathrm{~s}$-resulting in a layer thickness of upto $100 \mu \mathrm{m}$. This speed and time of the spin process were selected by trial and error. Higher speeds resulted in the thinner films which could not be polarized under an electric field. Shorter spin times produced samples of thicker section which as a result inhibited the complete volumetric cure of the samples. Polymerization of the thin film piezocomposite samples was undertaken using an Intertronics 
IUV250 Hand Lamp (Intertronics, Oxfordshire, England). Prior to curing, the samples were placed into an air-tight zip-lock bag, the air was expunged from the bag using nitrogen gas-to solve the problem of oxygen inhibition which occurred during the polymerization [20] of the composite material while under the UV light. Finally, silver paint (SigmaAldrich Co Ltd, Dorset, UK) was coated on the both surface of the sample as the top electrode and bottom electrode before the polarization process.

The completed sample was then poled using the direct method as shown in Fig. 2. This process involved applying an electric field (E) to cause the BT electric charges which (hitherto were randomly arranged within the NOA 65 chains after the cure process) to reorient themselves in the direction of applied field. Thus, each sample was placed in silicon oil to prevent surface discharges and heated to a constant temperature of $100{ }^{\circ} \mathrm{C}$. A voltage of $1.5 \mathrm{kV}$ was simultaneously applied across the surface of the samples for $90 \mathrm{~min}$. Although the samples were also poled under higher fields up to $5 \mathrm{kV}$, this led to the electric breakdown of the samples. The breakdown voltage was found to be around $2 \mathrm{kV}$. The dielectric strength of the cured piezocomposites samples were found to be independent on the particle sizes and determined as $20 \mathrm{MV} / \mathrm{m}$ for the sample thickness of $100 \mu \mathrm{m}$. While cured samples consisting of only NOA 65 had a dielectric strength of $<1 \mathrm{MV} / \mathrm{m}$. Electrical connections were made using 24 AWG gauge copper wires connected to both sides of each sample after the polarization process.

\subsection{Structural analysis}

The microstructure of three different samples containing BT nanoparticle sizes $(100 \mathrm{~nm}, 200 \mathrm{~nm}$ and

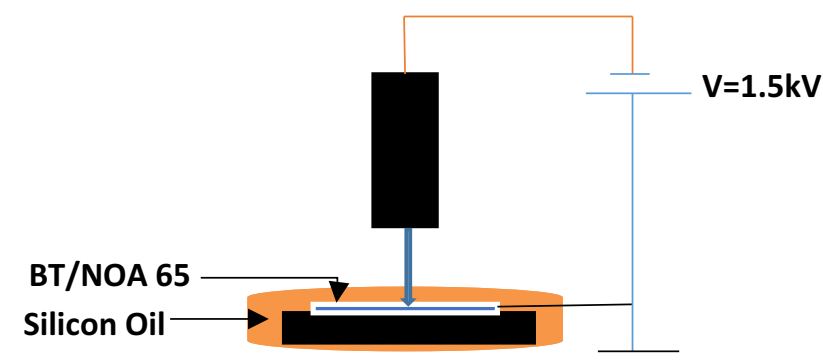

Fig. 2 Shows the method used to polarize the piezocomposite material. Each thin film sample was sandwiched between a pin electrode and a base plate electrode and immersed in silicon oil raised to a temperature of $100{ }^{\circ} \mathrm{C}$. A poling voltage of $1.5 \mathrm{kV}$ was applied across each sample
$500 \mathrm{~nm}$ ) was investigated using a table-top scanning electron microscope (Hitachi TM1000, Krefeld, Germany) at an accelerating voltage of $15 \mathrm{kV}$. The microstructure of the (0-3) piezocomposite was investigated to determine the degree of dispersion between the different filler sizes and matrix since this greatly affects the poling result and thus piezoelectric and mechanical properties of the composites [21]. Prior to SEM analysis, all samples had to be coated with a thin layer of platinum under vacuum to obtain clear micrographs.

\subsection{FTIR analysis}

FTIR spectra of the composite samples were obtained using a Tensor II Bench ATR-IR (Bruker AXS, Coventry, United Kingdom); each spectrum was background subtracted. The samples were scanned in an inert atmosphere over a wave number range of $4000-400 \mathrm{~cm}^{-1}$ with a resolution better than $0.01 \mathrm{~cm}^{-1}$. FTIR spectroscopy is a complex method, which is especially useful since the resulting spectrum acts as a fingerprint for compounds. In this work, the FTIR analysis was performed to ascertain if different BT nanoparticle sizes and NOA 65 cause changes in the resultant FTIR spectra. Each sample consists of NOA 65 mixed with BT in a ratio 40:60, respectively. BT particles have three different sizes (100, 200 and $500 \mathrm{~nm})$ and the combinations are reported in Fig. $4 a-c$, respectively.

\subsection{Mechanical properties}

The mechanical properties of piezocomposites depend strongly on the adhesion between the filler and matrix, the filler size, and filler loading [22]. With smaller particle sizes providing higher strength, higher rigidity, increased tensile strength [23, 24]. The effect of the particle sizes on the Indentation modulus of the samples was evaluated using a nanoindenter (Oxford Instruments Asylum Research, MFP-3D-BIO, Santa Barbara, CA, USA) with a calibrated Berkovich tip made of single crystalline diamond and results shown in Table 2. Analysis was carried out using the Oliver and Pharr method [25] to determine the average indentation modulus (E) of each sample. When this method is used the mechanical properties of the piezocomposite (hardness and elastic modulus) is determined from indentation load-displacement data obtained during cycle of loading and 
unloading [25]. Measurement of different samples consisting of only cured NOA 65, and piezocomposites with different nanoparticles sizes were taken in $6 \times 6$ array indentations, separated by $15 \mu \mathrm{m}$ in both axis was carried out to a fixed depth of $1 \mu \mathrm{m}$ in order to reduce the influence from neighboring indentations and to avoid the substrates influence on the mechanical properties. A maximum load of $460 \mu \mathrm{N}$ was applied.

\subsection{Piezoelectric properties}

The average longitudinal piezoelectric coefficient of the (0-3) piezocomposites was investigated using a microscanning laser doppler vibrometer technique $[26,27]$ employing a Polytec MSA100-3D (Polytec, Waldbronn, Germany). This method is based on the converse piezoelectric principle. Each sample is firmly glued onto glass slides using general purpose super glue and placed on a steel block $3 \mathrm{~cm}$ by $3 \mathrm{~cm}$ to eliminate bending of the substrate. The voltage stimulus was generated by the MSA100-3D internal data acquisition board of the laser vibrometer. A sinusoidal AC voltage of $10 \mathrm{~V}$ was applied across the axis of charging and the resulting displacement along the poling axis was measured. A focused image of the sample is first obtained and a frequency sweep stimulus was applied from 1 to $20 \mathrm{kHz}$ to obtain the resonant frequency of each sample after which measurements are obtained below the resonant frequency. The laser is focused at the centre of each sample and single point scans are obtained. A more detailed surface scan of each sample with a total of 1430 scan points were now taken to obtain the average $d_{33}$. All measurements were averaged over 20 cycles, using complex averaging.

\section{Results and discussion}

\subsection{Morphology}

The degree of homogeneity of the filler and matrix was observed as shown in Fig. 3. The micrograph confirms the (0-3) structure of the composite for each of the sample since the particles all appear to be unconnected with the polymer matrix. Furthermore, the degree of dispersion of the BT filler material at different particle sizes in the matrix was also observed. Figure 3a shows the case for BT (100 nm).
Here, the filler is seen to be densely and uniformly populated within the matrix at both levels of magnification. Figure $3 b$ and $c$ shows the case for BT $(200 \mathrm{~nm})$ and BT $(500 \mathrm{~nm})$. The same uniform distribution can be observed here and at higher magnification, the filler appears more homogenous with no apparent agglomeration. Although, some spaces were observed (white arrows) in composites with higher particle sizes (200 and $500 \mathrm{~nm}$ ), indicating that the thickness of the NOA 65 matrix surrounding the BT nanoparticles increases with an increase in BT particles sizes. It can be concluded from these SEM micrographs that a $(0-3)$ piezocomposite structure can be obtained using different particles sizes of BT and NOA 65. During the solution mixing, it is possible for agglomeration of the BT nanoparticles to occur due to the attracting van der waal forces between the nanoparticles.

This agglomeration of the BT $(500 \mathrm{~nm})$ nanoparticles was observed in [28], however, the ball milling technique used when mixing the piezocomposites has deagglomerated the BT nanoparticles thereby producing a (0-3) piezocomposite with uniform dispersion. Several studies have also shown that the deagglomeration of the nanoparticles can be obtained through particle coating, the use of surfactants, or charging the nanoparticle surface to repel them [29]. However, these methods tend to degrade the properties of the nanocomposites.

\subsection{FTIR analysis}

The polymer matrix NOA 65 contains in its structure a N-H group, which is visible in the FTIR spectra in the band $3450-3330 \mathrm{~cm}^{-1}$ while in the band $2900-2800 \mathrm{~cm}^{-1}$ the peaks indicate aliphatic groups $\mathrm{CH}_{3}$ and $\mathrm{CH}_{2}$ [25]. These characteristic peaks are present in spectra in Fig. 4. When NOA 65 is combined with BT, the obtained results show how the main difference in the structure is detectable only in the fingerprint region $\left(1500-500 \mathrm{~cm}^{-1}\right)$. In this region, it is much more difficult to pick out individual bonds than it is in the "cleaner" region at higher wavenumbers. The changes in the fingerprint region indicate the bonds between NOA 65 and BT particles.

One of these bonds could be $\mathrm{Ti}-\mathrm{O}$ but it is not easily discernable. Further analysis using NMR, or TOF-SIMS would be needed here. However, extra analysis with those techniques results in further complications due to the opaque nature of the 
Fig. 3 Representative scanning electron micrographs of the post cured (0-3)

Piezocomposite. a Shows the surface area of the piezocomposite containing BT nanoparticles $(100 \mathrm{~nm})$ at 60 $\mathrm{wt} \%$. $\mathbf{b}$ Shows the surface area of the piezocomposite containing BT nanoparticles $(200 \mathrm{~nm})$ at $60 \mathrm{wt} \%$. c Shows the surface area of the piezocomposite containing $\mathrm{BT}$ nanoparticles $(500 \mathrm{~nm})$ at 60 wt $\%$. BT nanoparticle was densely packed and appeared to be uniformly distributed across all the sample. The composite material had a thickness of $100 \mu \mathrm{m}$ (a)
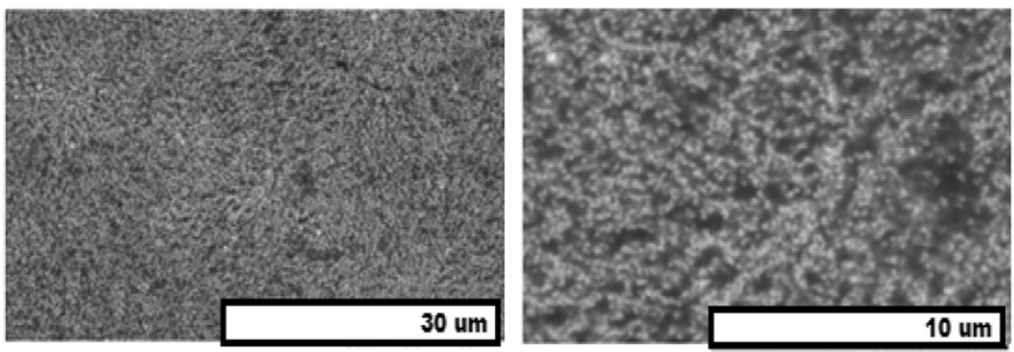

(b)
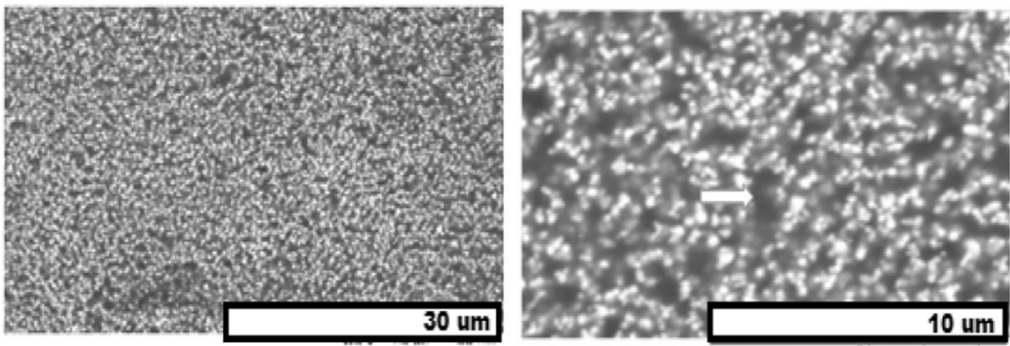

(c)

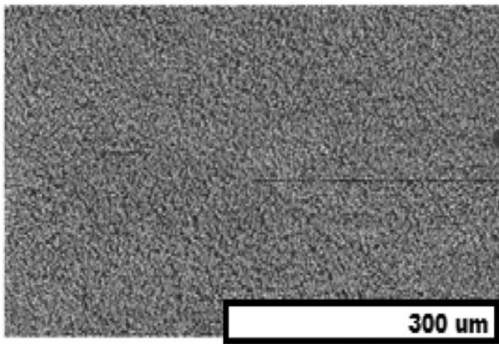

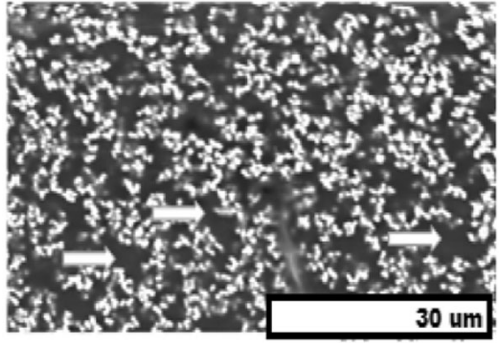

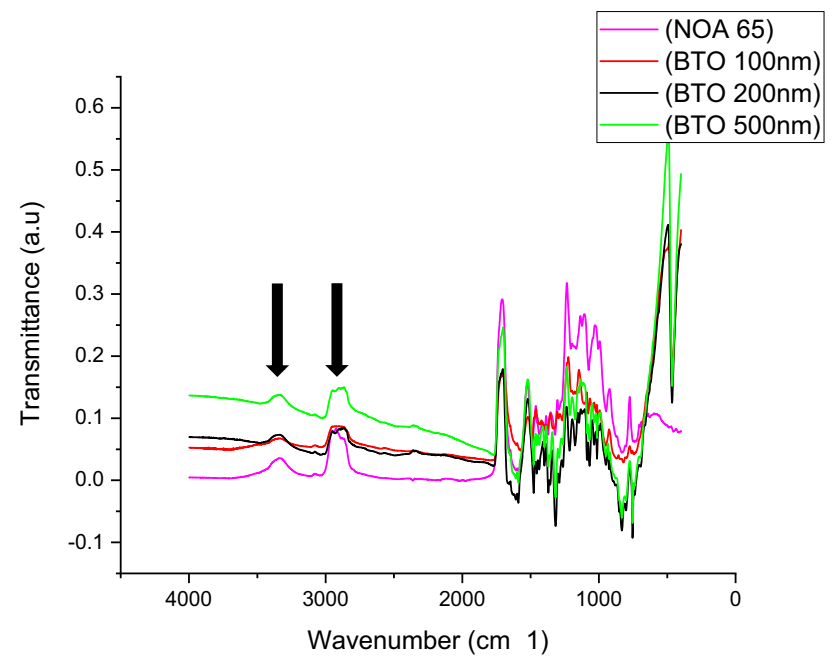

Fig. 4 FTIR spectra for a mixture of BT with different sizes and NOA 65. a The red spectra shows a combination of $60 \%$ of BT with a size of $100 \mathrm{~nm}$ and $40 \%$ of NOA 65 . b The black spectra indicates $60 \%$ of BT with a size of $200 \mathrm{~nm}$ and $40 \%$ of NOA 65 . c The green spectra corresponds to a combination of $60 \%$ of BT with a size of $500 \mathrm{~nm}$ and $40 \%$ of NOA 65 . $\mathrm{d}$ The purple spectra is the signal for pure NOA 65. All spectra are detected from 4000 to $400 \mathrm{~cm}^{-1}$. The arrows indicate the two recurrent peaks that characterize the consistent structure of NOA 65 (Color figure online) polymer. Depending on BT particle size, the combination with NOA 65 produces a different pattern of troughs in the spectrum.

Despite the fingerprint region changes, the rest of the FTIR spectra looks quite similar when BT with varying particle sizes are added. This result indicates that the combination between the polymer and the ferroelectric material does not alter the native structure of NOA 65.

\subsection{Piezoelectric properties}

The $d_{33}$ coefficient of the (0-3) piezocomposite consisting of varying filler particle sizes was investigated using the direct displacement technique with a laser vibrometer. The surface displacement as a function of frequency during single point scans is shown in Fig. 5. The mechanical displacement of each piezocomposite is observed with the BT $(500 \mathrm{~nm})$ having maximum displacement at $14 \mathrm{kHz}, \mathrm{BT}(200 \mathrm{~nm})$ at $9 \mathrm{kHz}$ and BT $(100 \mathrm{~nm})$ at $10 \mathrm{kHz}$. The average $d_{33}$ measured for each sample using the laser vibrometer is shown in Figs. 6 and 7. A full surface scan with a grid of 1430 scan points is performed at each resonant 


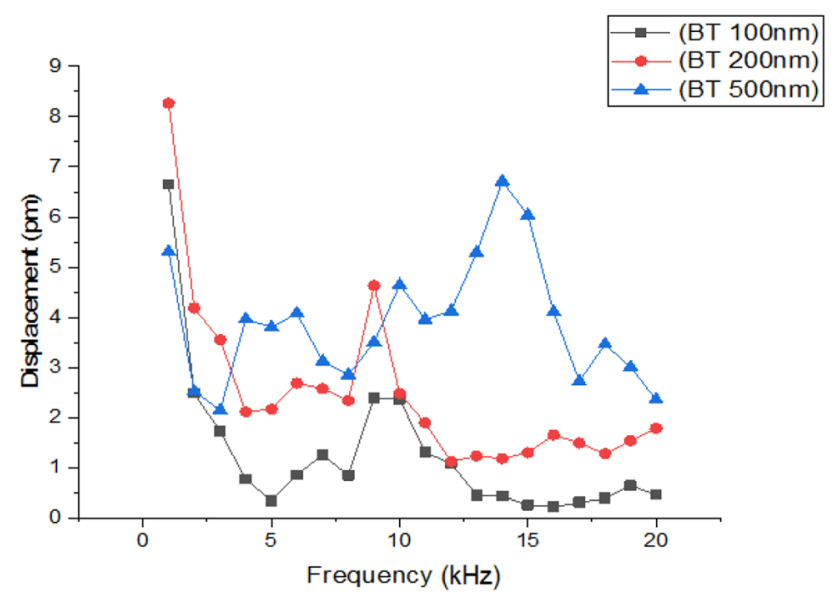

Fig. 5 Shows the result obtained during single point scans. It shows the magnitude of mechanical displacement as a function of a driving sinusoidal voltage of $10 \mathrm{~V} \mathrm{p}-\mathrm{p}$ delivered over a frequency range between 1 and $20 \mathrm{kHz}$

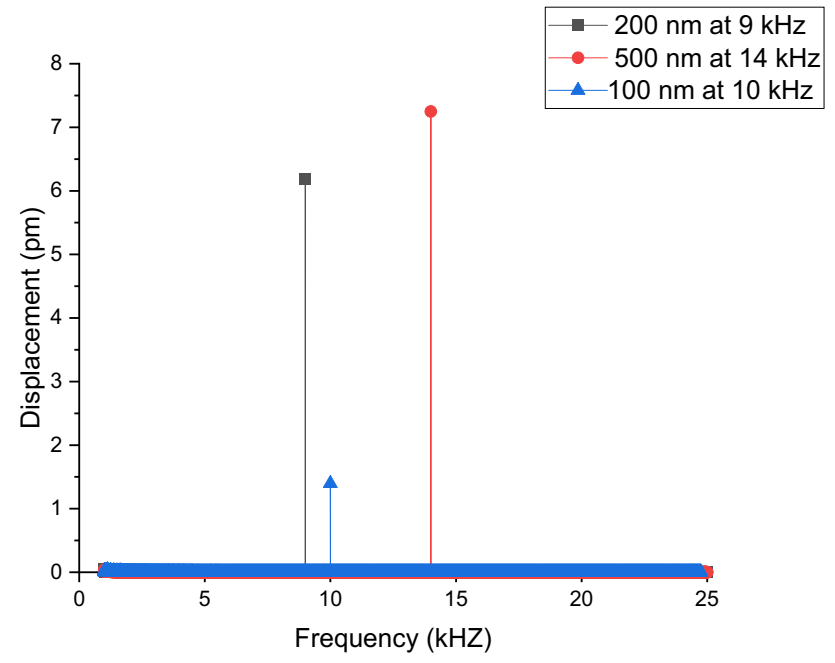

Fig. 6 Shows the average $d_{33}$ of each sample at the peak frequencies over 1430 scan points. As a function of a driving sinusoidal voltage of $10 \mathrm{~V} \mathrm{p}-\mathrm{p}$ delivered over a frequency range between 1 and $25 \mathrm{kHz}$

frequency obtained during the single point scan, a good signal to noise ratio was observed with the noise floor ranging less than $1 \mathrm{pm}$ across the scanned frequency as shown in Fig. 6. This could be as a result of the excellent optical properties of NOA 65 and the thickness of the composites. It was also observed that the $d_{33}$ increases with increase in BT particle sizes. This may be due to the effect of poling on the (0-3) piezocomposite. Previous work observed a transition from single domain to multi domain in BT/PVDF composites with BT particle sizes greater than

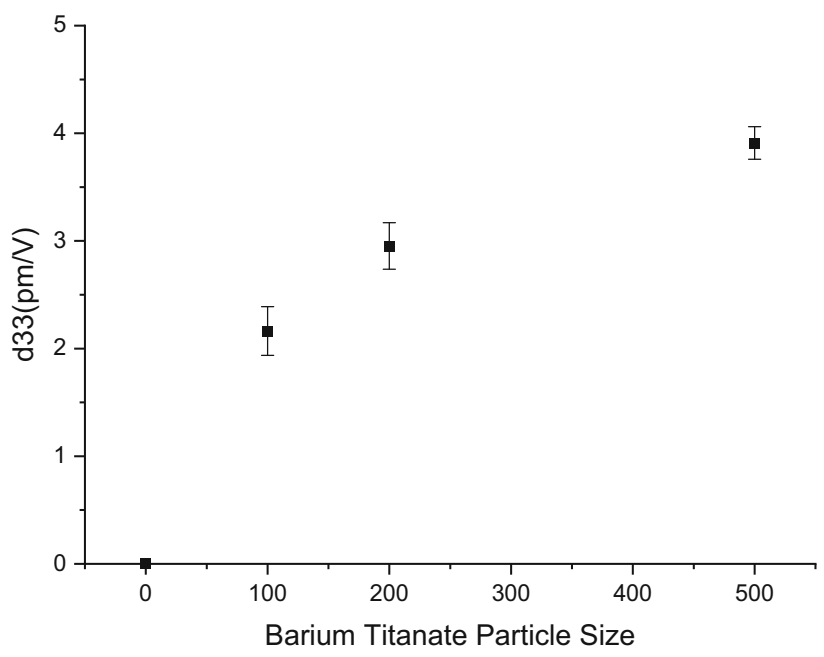

Fig. 7 Shows the average $d_{33}$ of obtained from 3 samples each over a frequency range of 3 and $13 \mathrm{kHz}$. The effect of nanoparticle sizes on the $d_{33}$ can be observed. The $d_{33}$ increases with an increase in the particle sizes

$100 \mathrm{~nm}$ [30]. Furthermore, it has been reported that the size of the BT crystal determines the phase transition and domain configuration of BT powder [30]. Although each composite had BT $60 \mathrm{wt} \%$, the composite with higher BT nanoparticles size may require less activation energy for domain orientation.

The piezoelectric property of the samples based on the direct piezoelectric principle was obtained using a quasi-static piezoelectric ZJ-6B $d_{33} / d_{31}$ meter (Institute of Acoustics, Chinese Academy of Sciences, Beijing, China). This process has been used to determine the piezoelectric property of single crystals, ceramics and thin films [31] and involved applying a periodic force of $0.25 \mathrm{~N}$ to the sample and measuring the resulting charge generated by the sample. The average $d_{33}$ constant measured is compared in Table 1. Although the measured values obtained using the LDV technique and the $d_{33}$ meter were quite different, measuring the $d_{33}$ coefficient of thin films using the $d_{33}$ meter is prone to error due to the bending effect of the thin film samples.

\subsection{Mechanical properties}

The effect of the BT nanoparticle sizes $(100 \mathrm{~nm}$, $200 \mathrm{~nm}$ and $500 \mathrm{~nm}$ ) on the mechanical properties of the samples was investigated using the method of Oliver and Pharr [25]. The indentation modulus $(n=36)$ obtained for each sample is shown in Table 2. It is seen that the values of $\mathrm{E}$ were found to decrease 
Table 1 Comparison of $d_{33}$ coefficient obtained from the LDV and $d_{33}$ meter

\begin{tabular}{llll}
\hline Sample & LDV $(\mathrm{pm} / \mathrm{V})$ single point scan & LDV $(\mathrm{pm} / \mathrm{V})$ full surface scan (average) & $d_{33}$ meter $(\mathrm{pC} / \mathrm{N})$ \\
\hline NOA 65:BT $(100 \mathrm{~nm})$ & 2.2 & 1.4 & 1.1 \\
NOA 65:BT $(200 \mathrm{~nm})$ & 2.9 & 6.1 & 2.1 \\
NOA 65:BT $(500 \mathrm{~nm})$ & 3.9 & 7.2 & 3.6 \\
\hline
\end{tabular}

Table 2 Values of Indentation modulus obtained to determine the effect of the BT nanoparticle sizes

\begin{tabular}{ll}
\hline Sample & Indentation modulus (Mpa) \\
\hline NOA 65 & 10.34 \\
NOA 65:BT $(100 \mathrm{~nm})$ & $31 \pm 10$ \\
NOA 65:BT $(200 \mathrm{~nm})$ & $22 \pm 4$ \\
NOA 65:BT $(500 \mathrm{~nm})$ & $20 \pm 2$ \\
\hline
\end{tabular}

significantly with an increase in particle size, although each sample had the same wt\% loading, this result may be attributed to the agglomeration of the filler and the high filler ratio used. Some studies have shown that particle sizes in the range of 1-62 $\mu \mathrm{m}$ tends to have little or no effect on the indentation modulus, and for lower volume fractions (10-18 vol\%). However, for higher volume fractions (30-46 vol\%) there is a slight decrease in modulus with increasing particle sizes [32, 33].

\section{Conclusions}

A piezocomposite material with a (0-3) structure was developed using BT nanoparticles and an ultraviolet curable polymer NOA 65 . The direct and inverse piezoelectric property of the material was examined using both the LDV technique and the $d_{33}$ meter, the average $d_{33}$ coefficient obtained from the LDV (full surface scan) showed that the $d_{33}$ increases with increase in particle size and further predicts a better measurement technique since it eliminates the bending effect of the thin film which occurs when testing the $d_{33}$ of thin films using the $d_{33}$ meter. The measured $d_{33}$ values of $2-7 \mathrm{pm} / \mathrm{V}$ shows some improvement over that of BT composites consisting of Photocurable polymers such as PEGDA and BEMA. Although each sample tested had maximum displacement at different regions of the bulk of the material, which may be due to the agglomeration of the filler nanoparticles and the method of poling. The 3D LDV single point experiments showed that at higher frequencies $(9 \mathrm{kHz}$ for the $100 \mathrm{~nm}$ sample, $10 \mathrm{kHz}$ for the $200 \mathrm{~nm}$ sample, and $14 \mathrm{kHz}$ for the $500 \mathrm{~nm}$ sample) all the samples vibrate with higher amplitudes which could be due to the samples having a uniform thickness and homogeneity across different regions. This property suggests the possibility of using this novel piezocomposite as a functional material for 3D-printed piezoelectric devices such as the devices developed in [8, 9]. The mechanical properties of the composite material were tested using the nanoindentation method, whereby the load-displacement curve and values obtained showed that the material exhibits plastic behavior and the indentation modulus decreases with increasing particle size. This effect is due to high BT loading and the size of the BT nanoparticles employed in fabricating the samples since the BT nanoparticles have a larger modulus than the matrix [34]. The SEM analysis of the composite material showed a uniform distribution of BT nanoparticles within the matrix and good bonding condition irrespective of the filler particle size used. Further work is required investigate the effect of filler/matrix adhesion, filler loading on the composite material stiffness and strength and the effect of different particle sizes on the dielectric properties. Ways of enhancing the $d_{33}$ value of this new piezocomposite and 3D printing of structures and devices with this material needs to be explored.

\section{Acknowledgements}

The research work was supported by the Engineering and Physical Sciences Research Council (EPSRC) under Grant EP/L022125/1, and by the European Research Council under the European Union's Seventh Framework Programme (FP/2007-2013) / ERC Grant Agreement n. [615030]. It was partially 
funded by the REACH HIGH Scholars ProgramPost-Doctoral Grants. The grant is part-financed by the European Union, Operational Program II, Cohesion Policy 2014-2020 (Investing in human capital to create more opportunities and promote the wellbeing of society European Social Fund).

Open Access This article is licensed under a Creative Commons Attribution 4.0 International License, which permits use, sharing, adaptation, distribution and reproduction in any medium or format, as long as you give appropriate credit to the original author(s) and the source, provide a link to the Creative Commons licence, and indicate if changes were made. The images or other third party material in this article are included in the article's Creative Commons licence, unless indicated otherwise in a credit line to the material. If material is not included in the article's Creative Commons licence and your intended use is not permitted by statutory regulation or exceeds the permitted use, you will need to obtain permission directly from the copyright holder. To view a copy of this licence, visit http://creativecommons.org/licen ses/by/4.0/.

Supplementary Information: The online version containssupplementary material available at http s://doi.org/10.1007/s10854-021-05818-5.

\section{References}

1. D.I. Woodward, C.P. Purssell, D.R. Billson, D.A. Hutchins, S.J. Leigh, Additively-manufactured piezoelectric devices. Phys. Status Solidi A 212, 2107-2113 (2015)

2. K.H. Han, R.E. Riman, A. Safari, Dielectric and piezoelectric properties of flexible 0-3 piezoelectric composites prepared by coprecipitated ( $\mathrm{Pb} / \mathrm{sub} 0.5 / \mathrm{Bi} / \mathrm{sub} 0.5 /)(\mathrm{Ti} / \mathrm{sub} 0.5 /(\mathrm{Fe} / \mathrm{sub}$ $1-\mathrm{x} / \mathrm{MN} / \mathrm{sub} \mathrm{x} / \mathrm{)} / \mathrm{sub} 0.5 /) \mathrm{O} / \mathrm{sub} 3 /(\mathrm{x}=0.00-0.02)$ ceramic powders. in Proceedings 1990 IEEE 7th International Symposium on Applications of Ferroelectrics, Urbana-Champaign, IL, USA, 1990, pp. 591-594. https://doi.org/10.1109/ ISAF.1990.200322

3. E.K. Akdogan, M. Allahverdi, A. Safari, Piezoelectric composites for sensor and actuator applications, member. IEEE Trans. Ultrason. Ferroelectr. Freq. Control 52(5), 746 (2005)

4. L. Jogschies, D. Klaas, R. Kruppe, J. Rittinger, P. Taptimthong, A. Wienecke, L. Rissing, M.C. Wurz, Recent developments of magnetoresistive sensors for industrial applications. Sensors 15, 28665-28689 (2015). https://doi.or $\mathrm{g} / 10.3390 / \mathrm{s} 151128665$

5. D. Thuau, M. Abbas, G. Wantz, L. Hirsch, I. Dufour, C. Ayela, Piezoelectric polymer gated OFET: cutting-edge electro-mechanical transducer for organic MEMS-based sensors. Sci. Rep. 6, 38672 (2016). https://doi.org/10.1038/srep3867 2PMCID:PMC5141423

6. M.D. Williams, B.A. Griffin, T.N. Reagan, J.R. Underbrink, M. Sheplak, AnAlN MEMS piezoelectric microphone for aeroacoustic applications. J. Microelectromech. Syst. 21(2), 270-283 (2012)

7. J.B. Park, A.F. von Recum, G.H. Kenner, B.J. Kelly, W.W. Coffeen, M.F. Grether, Piezoelectric ceramic implants: a feasibility study. J. Biomed. Mater. Res. 14, 269-277 (1980)

8. B. Tiller, A. Reid, B. Zhu, J. Guerreiro, R. Domingo-Roca, J.C. Jackson, J.F. Windmill, Piezoelectric microphone via a digital light processing 3D printing process. Mater. Des. 165, 107593 (2019)

9. R. Domingo-Roca, J.C. Jackson, J.F. Windmill, Bioinspired 3D-printed piezoelectric device for acoustic frequency separation. Sens. Actuator A Phys. 271, 1-8 (2017). https://doi. org/10.1109/ICSENS.2017.8234384

10. K. Kim, W. Zhu, X. Qu, C. Aaronson, W.R. McCall, S. Chen, D.J. Sirbuly, 3D optical printing of piezoelectric nanoparticlepolymer composite materials. ACS Nano 8, 9799-9806 (2014)

11. J. Feng, H. Yuan, X. Zhang, Promotion of osteogenesis by a piezoelectric biological ceramic. Biomaterials 18, 1531-1534 (1997)

12. X.-D. Chen, D.-B. Yang, Y.-D. Jiang, Wu. Zhi-Ming, D. Li, F.-J. Gou, J.-D. Yang, 0-3 Piezoelectric composite film with high d33 coefficient. Sens. Actuators A 65, 194-196 (1998)

13. Z. Chen, X. Song, L. Lei, X. Chen, C. Fei, C.T. Chiu, X. Qian, T. Ma, Y. Yang, K. Shung, Y. Chen, Q. Zhou, 3D printing of piezoelectric element for energy focusing and ultrasonic sensing. Nano Energy 27, 78-86 (2016)

14. K.J. Baeg, M. Caironi, Y.Y. Noh, Toward printed integrated circuits based on unipolar or ambipolar polymer semiconductors. Adv. Mater. 25, 4210-4244 (2013)

15 B. Pinto-Iguanero, A. Olivares-Pérez, I. Fuentes-Tapia, Holographic material film composed by NorlandNoa $65 \AA$ adhesive. Opt. Mater. 20(3), 225-232 (2002). https://doi.org/ 10.1016/S0925-3467(02)00068-X

16. J.Y. Na, B. Kang, D.H. Sin, K. Cho, Y. Don, Park effects of spin-coating time and processing additives. Sci. Rep. 5, 13288 (2015). https://doi.org/10.1038/srep13288

17. B. Kang, W.H. Lee, K. Cho, Recent advances in organic transistor printing processes. ACS Appl. Mater. Intefaces 5, 2302-2315 (2013) 
18. M. Campoy-Quiles et al., Morphology evolution via self-organization and lateral and vertical diffusion in polymer: fullerene solar cell blends. Nat. Mater. 7, 158-164 (2008)

19. J.Y. Na, B. Kang, D.H. Sin, K. Cho, Y. Don, Park understanding solidification of polythiophene thin films during spin-coating: effects of spin-coating time and processing additives. Sci. Rep. 5, 13288 (2015). https://doi.org/10.1038/ srep 13288

20. S.C. Ligon, B. Husár, H. Wutzel, R. Holman, R. Liska, Strategies to reduce oxygen inhibition in photoinduced polymerization. Chem. Rev. 114, 557-589 (2014)

21. S. Kim et al., Sequential solvent casting for improving the structural ordering and electrical characteristics of polythiophene thin films. RSC Adv. 4, 41159-41163 (2014)

22. Y. Nakamura, M. Yamaguchi, M. Okubo, T. Matsumoto, Effect of particle size on mechanical properties of epoxy resin filled withangular-shaped silica. J. Appl. Polym. Sci. 44, 151-158 (1992)

23. A.C. Roulin-Moloney, W.J. Cantwell, H.H. Kausch, Parametersdetermining the strength and toughness of particulatefilled epoxy resins. Polym. Compos. 8, 314-323 (1987)

24. N. Suzuki, M. Osada, M. Billah, Y. Bando, Y. Yamauchi, S.A. Hossain, Chemical synthesis of porous barium titanate thin film and thermal stabilization of ferroelectric phase by porosity-induced strain. J. Vis. Exp. (2018). https://doi.org/ $10.3791 / 57441$

25. W.C. Oliver, G.M. Pharr, An improved techique for determinining hardness and elastic modulus using load and displacement sensing indentation experiments. J. Mater. Res. 7, 1564-1583 (1992)

26. K. Yao, F.E.H. Tay, Measurement of longitudinal piezoelectric coefficient of thin films by a laser-scanning vibrometer.
IEEE Trans. Ultrason. Ferroelectr. Freq. Control 50, 113-116 (2003)

27. J. Hernando, J.L. Sánchez-Rojas, S. González-Castilla, E. Iborra, A. Ababneh, U. Schmid, Simulation and laser vibrometry characterization of piezoelectric AlN thin films. J. Appl. Phys. 104, 053502 (2008)

28. J. Li et al., A stable solution-processed polymer semiconductor with record high-mobility for printed transistors. Sci. Rep. 2, 754-762 (2012)

29. H. Sirringhaus et al., Two-dimensional charge transport in self-organized, high-mobility conjugated polymers. Nature 401, 685-688 (1999)

30 Á.S. de Inestrillas, F. Camarena, M.B. Cabo, J.M. Barreiro, A. Reig, Design and performance of a metal-shielded piezoelectric sensor. Sensors (Basel) 17(6), 1284 (2017). https://doi.org/ $10.3390 / \mathrm{s} 17061284$

31. R.Y. Ting, Composite piezoelectric materials for transduction. Appl. Acoust. 41, 325-335 (1994)

32. H.-I. Hsiang, F.-S. Kwo-Yin Lin, C.-Y. Yen, Effects of particle size of BaTiO3 powder on the dielectric properties of $\mathrm{BaTiO}$ /polyvinylidene fluoride composites. J. Mater. Sci. 36, 3809-3815 (2001)

33. S. Sahu, L.J. Broutman, Mechanical properties of particulate composites. Polym. Eng. Sci. 12, 91-100 (1972)

34. X. Song, Z. Chen, L. Lei, K. Shung, Q. Zhou, Y. Chen, Piezoelectric component fabrication using projection-based stereolithography of barium titanate ceramic suspensions. Rapid Prototyp. J. 23, 44-53 (2017)

Publisher's Note Springer Nature remains neutral with regard to jurisdictional claims in published maps and institutional affiliations. 